Editorial

\title{
Challenges and perspectives on the analysis of traditional perfluoroalkyl substances and emerging alternatives
}

Per- and polyfluoroalkyl substances (PFASs) are a group of anthropogenic additives with extensive industry and household applications since the 1950s. Particularly, perfluoroalkyl sulfonates and carboxylates have been subjected to public scrutiny, because of their ubiquitous presence in environmental compartments and biological species including human. In regard to the persistent, bioaccumulative and toxic properties, perfluorooctane sulfonic acid, its salts and perfluorooctane sulfonyl fluoride have been included in the Annex B of the Stockholm Convention on Persistent Organic Pollutants. The other (i.e. perfluorooctane carboxylic acid, perfluorohexane sulfonic acid) analogues and related compounds are also proposed as candidates to be listed under the convention. Restrictions on usages and voluntary phase-out initiatives of these PFASs are in progress, nevertheless, it is not the end of story. Fluorinated alternatives have been emerged in the marketplace in the recent decade, which would be inevitably released into the environment as well. Meanwhile, with the rapid development of modern instrumental techniques, more than a thousand kind of PFAS analytes are newly recognized, which could be artificial product ingredients, impurities or biological/abiotic environmental transformation products. These chemicals have large quantity in numbers and complexity in molecular structure, which bring more analytical issues on chromatograph separation, structure elucidation, accurate quantification, and biological effect assessment. The challenges make this special issue on "Analysis of Perfluoroalkyl Substances in the Environment and Human Health Samples" of great significance.

The topics in the special issue are diverse. Analytes of concern broadly covers fluorine speciation (e.g. inorganic fluorine, extractable organofluorine, unidentified organofluorine), traditional (e.g. $C \geq 8$ perfluoroalkyl carboxylic acid and sulfonic acid) and emerging (e.g. betaine-, chlorine-, and polyether-substituted analogues) PFAS compounds, and biological metabolites and intermediates. State-of-the-art methodologies are introduced, including passive sampling protocols for air and water, optimized pretreatments for varied matrixes (e.g. gas, aquatic, marine, solid, and biota), PFAS-featured nontarget identification, and data evaluation based on the progressive global interlaboratory assessments. Besides, approaches on generalized omic investigation and PFASprotein binding characterization were presented.

Titles of the selected 12 review papers are as follows:

[1] Towards a comprehensive analytical workflow for the chemical characterization of organofluorine in consumer products and environmental samples (Alina Koch, Leo W.Y. Yeung et al.)

[2] Analysis of emerging per- and polyfluoroalkyl substances: Progress and current issues (Yitao Pan, Jiayin Dai et al.)

[3] Is the phase-out of long-chain PFASs measureable as fingerprint in a defined area? Comparison of global PFAS concentrations and a monitoring study performed in Hesse, Germany from 2014 to 2018 (Raphael M. Janousek, Thomas P. Knepper et al.)

[4] Analysis of hexafluoropropylene oxide-dimer acid (HFPODA) by liquid chromatography-mass spectrometry (LC-MS): Review of current approaches and environmental levels (Lauren Mullin et al.)

[5] Unique analytical considerations for laboratory studies identifying metabolic products of per- and polyfluoroalkyl substances (Shira Joudan et al.)

[6] A critical review on passive sampling in air and water for perand polyfluoroalkyl substances (Foon Yin Lai, Lutz Ahrens et al.)

[7] Worldwide trends in tracing poly- and perfluoroalkyl substances (PFAS) in the environment (Shoji F. Nakayama et al.)

[8] Current analytical methodologies and gaps for per- and polyfluoroalkyl substances determination in the marine environment (Hongru Feng, Paul K.S. Lam et al.)

[9] High-resolution mass spectrometry (HRMS) methods for nontarget discovery and characterization of poly- and perfluoroalkyl substances (PFASs) in environmental and human samples (Yanna Liu, Guangbo Qu et al.)

[10] Global interlaboratory assessments of perfluoroalkyl substances under the Stockholm Convention on persistent organic pollutants ( $\mathrm{H}$. Fiedler et al.)

[11] An overview of omics approaches to characterize the effect of perfluoroalkyl substances in environmental health (Xinglei Yao, Maoyong Song et al.)

[12] Characterization of the binding of per- and poly-fluorinated substances to proteins: A methodological review (Xiaotu Liu, Da Chen et al.)

These review papers provide snapshots of current progress on analytical solution for the traditional PFAS pollutants and emerging alternatives. Knowledge gaps and future perspectives are further pointed out (for instance, include but not limited to): (1) insufficient data gained on precursor compounds and the air matrix; (2) limited attention focused on neutral volatile, and polyether PFAS analogues, which might call for the popularization of new instrumental techniques such as gas chromatograph coupled with high resolution 
mass spectrometry; (3) requirement of robust methods for elucidation the unresolved organofluorine components.

As guest editors of this special issue, we would like to thank all authors for their excellent contributions, all reviewers for the time and expertise advices. Special gratitude is also given to the journal publishing team for the support and effort in preparing the Special Issue.

Damià Barceló

Institute of Environmental Assessment and Water Research (IDAEA-

CSIC), Barcelona, Spain

E-mail address: dbcqam@idaea.csic.es.
Ting Ruan*

Research Center for Eco-Environmental Sciences, Chinese Academy of Sciences, Beijing, China

${ }^{*}$ Corresponding author. E-mail address: tingruan@rcees.ac.cn (T. Ruan).

Available online 26 July 2019 